\title{
Multilevel Gauss-Newton Methods for Phase Retrieval Problems
}

\author{
Birger Seifert ${ }^{1}$, Heinrich Stolz ${ }^{1}$, Marco Donatelli ${ }^{2}$, Dirk \\ Langemann $^{3}$, Manfred Tasche ${ }^{4}$ \\ ${ }^{1}$ Institut für Physik, Universität Rostock, D-18051 Rostock, Germany \\ ${ }^{2}$ Universitá dell'Insubria - Sede di Como, Dipartimento di Fisica e Matematica, Via \\ Valleggio 11, I-22100 Como, Italy \\ ${ }^{3}$ Institut für Mathematik, Universität zu Lübeck, Wallstr. 40, D-23560 Lübeck, \\ Germany \\ ${ }^{4}$ Institut für Mathematik, Universität Rostock, D-18051 Rostock, Germany \\ E-mail: birger.seifert@uni-rostock.de
}

\begin{abstract}
The phase retrieval problem is of wide interest because it appears in a number of interesting application areas in physics. Several kinds of phase retrieval problems appeared in laser optics over the past decade. In this paper we consider the numerical solution of two phase retrieval problems for an unknown smooth function $f$ with compact support. We approximate $f$ by a linear spline. The corresponding spline coefficients are iteratively determined by local Gauss-Newton methods, where convenient initial guesses are constructed by a multilevel strategy. We close with some numerical tests which illustrate our method.
\end{abstract}

PACS numbers: 02.60.Pn, 42.30.Rx, 42.65.Re 


\section{Introduction}

In many problems which arise in crystallography, electron microscopy, astronomy, coherence theory, and optics one often wishes to recover phase from only magnitude information simply because only the magnitude may be recorded or is available for measurement. For example, the so-called crystallographic phase problem is the problem of determining the phase information because in X-ray crystallography only the scattering intensity can be recorded, and the loss of the phase information makes the Fourier inversion impossible.

The optical phase problem recently appeared in current noninterferometric optical methods for the characterization of ultrashort optical signals. Noninterferometric spectrographic techniques like frequency-resolved optical gating (FROG) [1] or temporal analysis of spectral components (TASC) [2] rely on the solution of a phase retrieval problem. Only the magnitude of the Fourier transform of an electromagnetic wave is available for measurement. Therefore, the full characterization of the electromagnetic wave depends upon the retrieval of the phase from only spectral magnitude information. The ability to reconstruct the complex electric fields of ultrashort optical signals from only magnitude information is very useful in a number of current optical applications. The issue of the uniqueness of several schemes and methods suggested for FROG and TASC has been recently thoroughly analyzed [3]. But due to the well known stagnation problem, still a successful reconstruction by any of the known iterative algorithms [1] cannot be guaranteed.

The phase problem appears in one and higher dimensions. The results in [3] suggest to develop a working phase retrieval algorithm for the one-dimensional phase problem the more so as up to now no algorithm without stagnation problems for this case exist.

In a phase retrieval problem one seeks to recover an unknown compactly supported, sufficiently smooth function $f: \mathbb{R} \rightarrow \mathbb{C}$ from the modulus $|\hat{f}|$ of its Fourier transform [5]. In praxis, only values of $|\hat{f}|$ at finitely many equispaced nodes are given. Since phase and modulus of $f$ are, in general, independent of each other, it is necessary to use additional information on $f$. It should be stressed that the issue of the uniqueness is mentioned but not thoroughly analyzed in this paper. The purpose of this paper is to describe a new promising algorithm for the one-dimensional phase retrieval problem. We consider two one-dimensional phase retrieval problems which appear in several fields of mathematical physics.

In Section 2, we wish to recover an unknown compactly supported, sufficiently smooth function $f: \mathbb{R} \rightarrow \mathbb{C}$ although only finitely many, equispaced measurements of $|\hat{f}|$ and $|f|$ are given. Note that the recovery of $f$ is equivalent to the recovery of the phase of $f$. This kind of phase retrieval problem appears in electron microscopy, wave front sensing, and laser optics (e.g. FROG and TASC [3]). Our iterative algorithm for this phase retrieval problem is based on a linear B-spline approximation of $f$. The unknown complex spline coefficients are determined by a least squares problem which is iteratively solved by a local Gauss-Newton method. 
In Section 3, we consider a phase retrieval problem with non-negativity constraint and call it constrained phase retrieval problem. Here we reconstruct an unknown nonnegative, sufficiently smooth function $f: \mathbb{R} \rightarrow[0, \infty)$ with compact support, if finitely many equispaced measurements of $|\hat{f}|$ are given. This type of phase retrieval problem appears in image recovery from speckle interferometry data in astronomy and from structure factors in crystallography [4]. Our iterative algorithm for this constrained phase retrieval problem is again based on a linear B-spline approximation of $f$. The unknown non-negative spline coefficients are modeled by squares of real constants which are determined by a least squares problem without constraints. Then this least squares problem is solved by a local Gauss-Newton method.

The convergence of a local Gauss-Newton method depends on a good choice of an initial guess. In Section 4, we propose the construction of initial guesses by a multilevel strategy. Finally in Section 5, we present results of some numerical tests.

\section{Phase retrieval problem}

In electron microscopy, wave front sensing, and laser optics (e.g. FROG and TASC $[3])$, one often wishes to recover a compactly supported, sufficiently smooth function $f: \mathbb{R} \rightarrow \mathbb{C}$ although only finitely many equispaced measurements of $|f|$ and $|\hat{f}|$ are made. By $\hat{f}$ we denote the Fourier transform

$$
\hat{f}(v)=\int_{-\infty}^{\infty} f(x) \mathrm{e}^{-\mathrm{i} x v} \mathrm{~d} x \quad(v \in \mathbb{R}) .
$$

Assume that supp $f \subseteq[-1, N]$ is connected, where $N \in \mathbb{N}$ is some power of 2. Let $\varphi$ be the centered linear B-spline which is defined by

$$
\varphi(x):= \begin{cases}1+x & -1 \leq x<0 \\ 1-x & 0 \leq x \leq 1 \\ 0 & \text { otherwise }\end{cases}
$$

Then we get for the Fourier transform of $\varphi$ that

$$
\hat{\varphi}(v)=\left(\operatorname{sinc} \frac{v}{2}\right)^{2} \quad(v \in \mathbb{R})
$$

with

$$
\operatorname{sinc} v:= \begin{cases}\sin v / v & v \neq 0 \\ 1 & v=0 .\end{cases}
$$

For brevity, we denote the reconstructed function by $f$ too. In this section, we consider the following phase retrieval problem: Reconstruct the complex-valued linear spline function

$$
f(x)=\sum_{n=0}^{N-1} c_{n} \varphi(x-n) \quad\left(c_{n} \in \mathbb{C} ; x \in \mathbb{R}\right),
$$


if $|f(n)|(n=0, \ldots, N-1)$ and $\left|\hat{f}\left(\frac{k \pi}{N}\right)\right|(k=-N, \ldots, N-1)$ are given. Note that $\mathrm{e}^{\mathrm{i} \alpha} f$ with arbitrary $\alpha \in[0,2 \pi)$ is also a solution, if $f$ is a solution of this phase retrieval problem.

Using the properties of the Fourier transform, we obtain that

$$
\hat{f}(v)=\left(\sum_{n=0}^{N-1} c_{n} \mathrm{e}^{-\mathrm{i} n v}\right) \hat{\varphi}(v)
$$

and hence

$$
\overline{\hat{f}(v)}=\left(\sum_{n=0}^{N-1} \bar{c}_{n} \mathrm{e}^{\mathrm{i} n v}\right) \overline{\hat{\varphi}(v)}
$$

such that

$$
|\hat{f}(v)|^{2}=p(v)|\hat{\varphi}(v)|^{2}
$$

with

$$
p(v):=\left(\sum_{n=0}^{N-1} c_{n} \mathrm{e}^{-\mathrm{i} n v}\right)\left(\sum_{n=0}^{N-1} \bar{c}_{n} \mathrm{e}^{\mathrm{i} n v}\right) .
$$

The complex-valued trigonometric polynomial $p$ of order $N-1$ has the following form

$$
\begin{aligned}
p(v)= & \left(c_{0} \bar{c}_{0}+c_{1} \bar{c}_{1}+\ldots+c_{N-1} \bar{c}_{N-1}\right) \\
& +\left(c_{0} \bar{c}_{1}+c_{1} \bar{c}_{2}+\ldots+c_{N-2} \bar{c}_{N-1}\right) \mathrm{e}^{\mathrm{i} v} \\
& +\left(c_{0} \bar{c}_{2}+c_{1} \bar{c}_{3}+\ldots+c_{N-3} \bar{c}_{N-1}\right) \mathrm{e}^{2 \mathrm{i} v} \\
& \vdots \\
& +c_{0} \bar{c}_{N-1} \mathrm{e}^{(N-1) \mathrm{i} v} \\
& +\left(\bar{c}_{0} c_{1}+\bar{c}_{1} c_{2}+\ldots+\bar{c}_{N-2} c_{N-1}\right) \mathrm{e}^{-\mathrm{i} v} \\
& +\left(\bar{c}_{0} c_{2}+\bar{c}_{1} c_{3}+\ldots+\bar{c}_{N-3} c_{N-1}\right) \mathrm{e}^{-2 \mathrm{i} v} \\
& \vdots \\
& +\bar{c}_{0} c_{N-1} \mathrm{e}^{-(N-1) \mathrm{i} v} .
\end{aligned}
$$

Then we get that

$$
\frac{|\hat{f}(v)|^{2}}{|\hat{\varphi}(v)|^{2}}=\frac{|\hat{f}(v)|^{2}}{\left(\operatorname{sinc} \frac{v}{2}\right)^{4}}=p(v) \quad \text { for all } v \in[-\pi, \pi] .
$$

Now we choose $v_{k}=\frac{k \pi}{N}(k=-N, \ldots, N-1)$ and consider

$$
p\left(v_{k}\right)=d_{k}:=\frac{\left|\hat{f}\left(v_{k}\right)\right|^{2}}{\left(\operatorname{sinc} \frac{v_{k}}{2}\right)^{4}} \geq 0,
$$


i.e.

$$
\left(\mathrm{e}^{\pi \mathrm{i} j k / N}\right)_{j, k=-N}^{N-1}\left(\begin{array}{c}
0 \\
\bar{c}_{0} c_{N-1} \\
\vdots \\
\bar{c}_{0} c_{1}+\ldots+\bar{c}_{N-2} c_{N-1} \\
\left|c_{0}\right|^{2}+\ldots+\left|c_{N-1}\right|^{2} \\
c_{0} \bar{c}_{1}+\ldots+c_{N-2} \bar{c}_{N-1} \\
\vdots \\
c_{0} \bar{c}_{N-1}
\end{array}\right)=\left(d_{k}\right)_{k=-N}^{N-1}
$$

The coefficient matrix of this system of linear equations is the modified Fourier matrix of order $2 N$ :

$$
\left(\mathrm{e}^{\pi \mathrm{i} j k / N}\right)_{j, k=-N}^{N-1}
$$

Its inverse is the matrix

$$
\frac{1}{2 N}\left(\mathrm{e}^{-\pi \mathrm{i} j k / N}\right)_{j, k=-N}^{N-1} .
$$

The product of this matrix with the vector $\left(d_{k}\right)_{k=-N}^{N-1}$ can be computed by the fast Fourier transform of length $2 N$ (see [6], pp. 17-21) and is denoted by

$$
\left(e_{j}\right)_{j=-N}^{N-1}:=\frac{1}{2 N}\left(\mathrm{e}^{-\pi \mathrm{i} j k / N}\right)_{j, k=-N}^{N-1}\left(d_{k}\right)_{k=-N}^{N-1} .
$$

Note that $e_{-N}=0$. Thus we get the following system of nonlinear equations

$$
\begin{aligned}
\left|c_{0}\right|^{2}+\left|c_{1}\right|^{2}+\ldots+\left|c_{N-1}\right|^{2} & =e_{0}>0, \\
c_{0} \bar{c}_{1}+c_{1} \bar{c}_{2}+\ldots+c_{N-2} \bar{c}_{N-1} & =\frac{1}{2}\left(e_{1}+\bar{e}_{-1}\right) \in \mathbb{C}, \\
c_{0} \bar{c}_{2}+c_{1} \bar{c}_{3}+\ldots+c_{N-3} \bar{c}_{N-1} & =\frac{1}{2}\left(e_{2}+\bar{e}_{-2}\right) \in \mathbb{C}, \\
\vdots & \\
c_{0} \bar{c}_{N-1} & =\frac{1}{2}\left(e_{N-1}+\bar{e}_{1-N}\right) \in \mathbb{C} .
\end{aligned}
$$

From (3) it follows that

$$
e_{0}=\frac{1}{2 N} \sum_{k=-N}^{N-1} d_{k}
$$

By (1) we have $c_{n}=f(n)(n=0, \ldots, N-1)$ such that the relation

$$
\left|c_{0}\right|^{2}+\left|c_{1}\right|^{2}+\ldots+\left|c_{N-1}\right|^{2}=e_{0}
$$

can be considered as a discrete Parseval equation for the vectors $(f(n))_{n=0}^{N-1}$ and $\left(\hat{f}\left(v_{k}\right)\right)_{k=-N}^{N-1}$ :

$$
\sum_{n=0}^{N-1}|f(n)|^{2}=\frac{1}{2 N} \sum_{k=-N}^{N-1} \frac{\left|\hat{f}\left(v_{k}\right)\right|^{2}}{\left(\operatorname{sinc} \frac{v_{k}}{2}\right)^{4}} .
$$

In order to compute the complex coefficients $c_{n}(n=0, \ldots, N-1)$ of the function $f$, we have to solve the nonlinear system (4) under the restrictions $\left|c_{n}\right|^{2}=|f(n)|^{2}$ $(n=0, \ldots, N-1)$. Now we write this system (with complex unknowns $c_{n}$ ) as a system 
with real unknowns $a_{n}:=\operatorname{Re} c_{n}$ and $b_{n}:=\operatorname{Im} c_{n}(n=0, \ldots, N-1)$. Thus we obtain the following system

$$
\begin{aligned}
a_{0}^{2}+a_{1}^{2}+\ldots+a_{N-1}^{2}+b_{0}^{2}+b_{1}^{2}+\ldots+b_{N-1}^{2} & =h_{0}, \\
a_{0} a_{1}+a_{1} a_{2}+\ldots+a_{N-2} a_{N-1}+b_{0} b_{1}+b_{1} b_{2}+\ldots+b_{N-2} b_{N-1} & =h_{1}, \\
a_{0} a_{2}+a_{1} a_{3}+\ldots+a_{N-3} a_{N-1}+b_{0} b_{2}+b_{1} b_{3}+\ldots+b_{N-3} b_{N-1} & =h_{2}, \\
& \vdots \\
a_{0} a_{N-1}+b_{0} b_{N-1} & =h_{N-1}, \\
0 & =h_{N}, \\
b_{0} a_{1}+b_{1} a_{2}+\ldots+b_{N-2} a_{N-1}-a_{0} b_{1}-a_{1} b_{2}-\ldots-a_{N-2} b_{N-1} & =h_{N+1}, \\
b_{0} a_{2}+b_{1} a_{3}+\ldots+b_{N-3} a_{N-1}-a_{0} b_{2}-a_{1} b_{3}+\ldots-a_{N-3} b_{N-1} & =h_{N+2}, \\
& \vdots \\
b_{0} a_{N-1}-a_{0} b_{N-1} & =h_{2 N-1}
\end{aligned}
$$

under the constraints $a_{n}^{2}+b_{n}^{2}-|f(n)|^{2}=0(n=0, \ldots, N-1)$, where we set

$$
h_{n}:= \begin{cases}\frac{1}{2} \operatorname{Re}\left(e_{n}+\bar{e}_{-n}\right) & n=0, \ldots, N-1, \\ 0 & n=N \\ \frac{1}{2} \operatorname{Im}\left(e_{n-N}+\bar{e}_{N-n}\right) & n=N+1, \ldots, 2 N-1 .\end{cases}
$$

Let $\mathbf{a}:=\left(a_{n}\right)_{n=0}^{N-1}, \mathbf{b}:=\left(b_{n}\right)_{n=0}^{N-1}$ and $\mathbf{h}:=\left(h_{n}\right)_{n=0}^{2 N-1}$. Further we introduce the Hankel matrix $H(\mathbf{a})$ with the first row $\mathbf{a}^{\mathrm{T}}$, where below the counterdiagonal all elements are zero. Let $T(\mathbf{a})$ be the upper triangular Toeplitz matrix with the first row $\mathbf{a}^{\mathrm{T}}$. Then our nonlinear system reads as follows

$$
\left(\begin{array}{cc}
H(\mathbf{a}) & H(\mathbf{b}) \\
-H(\mathbf{b}) & H(\mathbf{a})
\end{array}\right)\left(\begin{array}{l}
\mathbf{a} \\
\mathbf{b}
\end{array}\right)-\mathbf{h}=\mathbf{0}
$$

subject to the nonlinear constraints

$$
\mathbf{a}^{2}+\mathbf{b}^{2}-\mathbf{f}=\mathbf{0}
$$

with $\mathbf{f}:=\left(|f(n)|^{2}\right)_{n=0}^{N-1}, \mathbf{a}^{2}:=\left(a_{n}^{2}\right)_{n=0}^{N-1}$ and $\mathbf{b}^{2}:=\left(b_{n}^{2}\right)_{n=0}^{N-1}$. Here $\mathbf{0}$ denotes a zero vector. Since the data vectors $\mathbf{h}$ and $\mathbf{f}$ are based on measurements, we consider instead of the above constrained nonlinear system the following unconstrained least squares problem with penalty parameter $\mu \geq 1$

$$
\left\|\left(\begin{array}{cc}
H(\mathbf{a}) & H(\mathbf{b}) \\
-H(\mathbf{b}) & H(\mathbf{a})
\end{array}\right)\left(\begin{array}{c}
\mathbf{a} \\
\mathbf{b}
\end{array}\right)-\mathbf{h}\right\|^{2}+\mu^{2}\left\|\mathbf{a}^{2}+\mathbf{b}^{2}-\mathbf{f}\right\|^{2}=\min .
$$

Here $\|\cdot\|$ denotes the Euclidean norm. The parameter $\mu$ balances the influence of the two nonlinear terms in (8). The second term in (8) is the constraint which is important for a good convergence of our iterative method. Hence we have $\mu \geq 1$. However $\mu \gg 1$ is not useful in general, since then the first nonlinear term in (8) is not sufficiently noticed. With the notations $\mathbf{y}:=\left(\mathbf{a}^{\mathrm{T}}, \mathbf{b}^{\mathrm{T}}\right)^{\mathrm{T}} \in \mathbb{R}^{2 N}, \mathbf{g}:=\left(\mathbf{h}^{\mathrm{T}}, \mathbf{f}^{\mathrm{T}}\right)^{\mathrm{T}} \in \mathbb{R}^{3 N}$, and

$$
F(\mathbf{y}):=G(\mathbf{y}) \mathbf{y}
$$


where $G(\mathbf{y})$ is the block matrix

$$
G(\mathbf{y}):=\left(\begin{array}{cc}
H(\mathbf{a}) & H(\mathbf{b}) \\
-H(\mathbf{b}) & H(\mathbf{a}) \\
\mu \operatorname{diag} \mathbf{a} & \mu \operatorname{diag} \mathbf{b}
\end{array}\right) \in \mathbb{R}^{3 N \times 2 N},
$$

which depends linearly on $\mathbf{y}$, the nonlinear system (6) under the constraints (7) is equivalent to the inverse problem

$$
F(\mathbf{y})=\mathbf{g} .
$$

Furthermore, the least squares problem (8) is equivalent to the Gauss-Newton problem

$$
\|F(\mathbf{y})-\mathbf{g}\|^{2}=\min .
$$

The inverse problem (10) is ill-posed. This means that (10) suffers from following deficiencies:

- (10) is not solvable for arbitrary given $\mathbf{g} \in \mathbb{R}^{3 N}$.

- If (10) is solvable, then its solution is not unique.

- A possible solution of (10) does not depend continuously on $\mathbf{g} \in \mathbb{R}^{3 N}$.

To cope with the ill-posedness, problem (10) has to be regularized. A known and effective technique is Tikhonov regularization [7]. We use an approximate minimizer of the Tikhonov functional

$$
\Phi(\mathbf{y}):=\|F(\mathbf{y})-\mathbf{g}\|^{2}+\alpha\|\mathbf{y}-\tilde{\mathbf{y}}\|^{2}
$$

as an approximate solution of the inverse problem (10), where $\alpha>0$ is a small regularization parameter and where $\tilde{\mathbf{y}} \in \mathbb{R}^{2 N}$ is a coarse approximation of the solution. The choice of $\tilde{\mathbf{y}}$ is crucial. Available a priori information about the location of least squares solution of (11) has to enter into the selection of $\tilde{\mathbf{y}}$. Later we will construct $\tilde{\mathbf{y}}$ by multilevel strategy. By the choice of $\tilde{\mathbf{y}}$, we can influence which least squares solution we want to approximate. It can be shown that the Tikhonov functional (12) is locally a convex functional.

Now we can explicitly compute the Jacobian as the block matrix

$$
F^{\prime}(\mathbf{y})=\left(\begin{array}{cc}
H(\mathbf{a})+T(\mathbf{a}) & H(\mathbf{b})+T(\mathbf{b}) \\
-H(\mathbf{b})+T(\mathbf{b}) & H(\mathbf{a})-T(\mathbf{a}) \\
2 \mu \operatorname{diag} \mathbf{a} & 2 \mu \operatorname{diag} \mathbf{b}
\end{array}\right) \in \mathbb{R}^{3 N \times 2 N}
$$

Thus $F^{\prime}(\mathbf{y})$ depends linearly on $\mathbf{y}$. In some sense, $F(\mathbf{y})$ is "quadratic", i.e., each component of $F(\mathbf{y})$ is a quadratic polynomial with $2 N$ variables. Under these conditions, there exists a vector $\mathbf{y}^{*} \in \mathbb{R}^{2 N}$ with

$$
\Phi\left(\mathbf{y}^{*}\right)=\min \left\{\Phi(\mathbf{y}) ; \mathbf{y} \in \mathbb{R}^{2 N}\right\} .
$$

Now we define a sequence $\left\{\mathbf{y}_{k}\right\}_{k=0}^{\infty}$ which converges to $\mathbf{y}^{*}$ for $k \rightarrow \infty$. Our algorithmic approach starts with the linear Taylor expansion

$$
F(\mathbf{y}) \approx F\left(\mathbf{y}_{k}\right)+F^{\prime}\left(\mathbf{y}_{k}\right)\left(\mathbf{y}-\mathbf{y}_{k}\right)
$$


Then the simplified Tikhonov functional

$$
\Phi^{(k)}(\mathbf{y}):=\left\|F\left(\mathbf{y}_{k}\right)+F^{\prime}\left(\mathbf{y}_{k}\right)\left(\mathbf{y}-\mathbf{y}_{k}\right)-\mathbf{g}\right\|^{2}+\alpha\|\mathbf{y}-\tilde{\mathbf{y}}\|^{2}
$$

is minimized with the vanishing gradient condition

$$
\nabla \Phi^{(k)}(\mathbf{y})=2 F^{\prime}\left(\mathbf{y}_{k}\right)^{\mathrm{T}}\left[F\left(\mathbf{y}_{k}\right)+F^{\prime}\left(\mathbf{y}_{k}\right)\left(\mathbf{y}-\mathbf{y}_{k}\right)-\mathbf{g}\right]+2 \alpha(\mathbf{y}-\tilde{\mathbf{y}})=\mathbf{o} .
$$

Thus we obtain the update formula

$$
\mathbf{y}_{k+1}=\mathbf{y}_{k}-\Delta \mathbf{y}_{k} \quad(k=0,1, \ldots)
$$

with

$$
\left[F^{\prime}\left(\mathbf{y}_{k}\right)^{\mathrm{T}} F^{\prime}\left(\mathbf{y}_{k}\right)+\alpha I\right] \Delta \mathbf{y}_{k}=F^{\prime}\left(\mathbf{y}_{k}\right)^{\mathrm{T}}\left(F\left(\mathbf{y}_{k}\right)-\mathbf{g}\right)+\alpha\left(\mathbf{y}_{k}-\tilde{\mathbf{y}}\right) .
$$

This Gauss-Newton method requires sufficiently good starting guesses $\mathbf{y}_{0}$, which we will obtain by a multilevel strategy in Section 4 . The linear system (15) has a unique solution, if a suitable regularization parameter $\alpha>0$ is chosen. Since the minimization of the Tikhonov functional (12) can be regarded as trust-region method, the linear system (15) uses the idea of the Levenberg-Marquardt method for (10), see [9], pp. $117-121$.

\section{Constrained phase retrieval problem}

Now we consider the problem of phase retrieval from a measurement of $|\hat{f}|$ and a non-negativity constraint $f \geq 0$. Such phase retrieval problems appear in image recovery from speckle interferometry data in astronomy and from structure factors in crystallography [4].

In this section, we consider the following constrained phase retrieval problem: Reconstruct the non-negative linear spline function

$$
f(x)=\sum_{n=0}^{N-1} c_{n}^{2} \varphi(x-n) \quad\left(c_{n}, x \in \mathbb{R}\right),
$$

if $\left|\hat{f}\left(\frac{k \pi}{N}\right)\right|(k=0, \ldots, N)$ are given. Here $\varphi$ denotes again the centered linear B-spline. The use of the squares $c_{n}^{2}$ assures the constraint $f \geq 0$, see [9], p. 188 .

Using the properties of the Fourier transform, we obtain that

$$
\hat{f}(v)=\left(\sum_{n=0}^{N-1} c_{n}^{2} \mathrm{e}^{-\mathrm{i} n v}\right) \hat{\varphi}(v)
$$

and hence

$$
\overline{\hat{f}(v)}=\left(\sum_{n=0}^{N-1} c_{n}^{2} \mathrm{e}^{\mathrm{i} n v}\right) \overline{\hat{\varphi}(v)}
$$

such that

$$
|\hat{f}(v)|^{2}=p(v)|\hat{\varphi}(v)|^{2}
$$


with

$$
p(v):=\left(\sum_{n=0}^{N-1} c_{n}^{2} \mathrm{e}^{-\mathrm{i} n v}\right)\left(\sum_{n=0}^{N-1} c_{n}^{2} \mathrm{e}^{\mathrm{i} n v}\right) .
$$

If (16) is a solution of the constrained phase retrieval problem, then

$$
g(x)=\sum_{n=0}^{N-1} c_{N-1-n}^{2} \varphi(x-n) \quad(x \in \mathbb{R}),
$$

is also a solution, since $g \geq 0$ and $\left|\hat{g}\left(\frac{k \pi}{N}\right)\right|=\left|\hat{f}\left(\frac{k \pi}{N}\right)\right|$ for all $k=0, \ldots, N$.

The real even trigonometric polynomial $p$ of order $N$ has the following form

$$
\begin{aligned}
p(v)= & \left(c_{0}^{4}+c_{1}^{4}+\ldots+c_{N-1}^{4}\right)+2\left(c_{0}^{2} c_{1}^{2}+c_{1}^{2} c_{2}^{2}+\ldots+c_{N-2}^{2} c_{N-1}^{2}\right) \cos v \\
& +2\left(c_{0}^{2} c_{2}^{2}+c_{1}^{2} c_{3}^{2}+\ldots+c_{N-3}^{2} c_{N-1}^{2}\right) \cos (2 v)+\ldots \\
& +2 c_{0}^{2} c_{N-1}^{2} \cos ((N-1) v) .
\end{aligned}
$$

Then we get that

$$
\frac{|\hat{f}(v)|^{2}}{|\hat{\varphi}(v)|^{2}}=\frac{|\hat{f}(v)|^{2}}{\left(\operatorname{sinc} \frac{v}{2}\right)^{4}}=p(v) \quad \text { for all } v \in[0, \pi] .
$$

Now we choose $v_{k}:=\frac{k \pi}{N}(k=0, \ldots, N)$ and consider

$$
p\left(v_{k}\right)=d_{k}:=\frac{\left|\hat{f}\left(v_{k}\right)\right|^{2}}{\left(\operatorname{sinc} \frac{v_{k}}{2}\right)^{4}} \geq 0 \quad(k=0, \ldots, N),
$$

i.e.

$$
2 C_{N+1}\left(\begin{array}{c}
c_{0}^{4}+c_{1}^{4}+\ldots+c_{N-1}^{4} \\
c_{0}^{2} c_{1}^{2}+c_{1}^{2} c_{2}^{2}+\ldots+c_{N-2}^{2} c_{N-1}^{2} \\
c_{0}^{2} c_{2}^{2}+c_{1}^{2} c_{3}^{2}+\ldots+c_{N-3}^{2} c_{N-1}^{2} \\
\vdots \\
c_{0}^{2} c_{N-1}^{2} \\
0
\end{array}\right)=\left(d_{k}\right)_{k=0}^{N}
$$

where we introduce the cosine matrix by

$$
C_{N+1}:=\left(\varepsilon_{k} \cos \frac{j k \pi}{N}\right)_{j, k=0}^{N}
$$

with $\varepsilon_{0}=\varepsilon_{N}:=\frac{1}{2}$ and $\varepsilon_{k}:=1$ for $k=1, \ldots, N-1$ (see [6], p. 229; [8]). The inverse matrix of $C_{N+1}$ is the scaled cosine matrix $\frac{2}{N} C_{N+1}$. Since $N$ is a power of 2 , the product of $C_{N+1}$ and the vector $\left(d_{k}\right)_{k=0}^{N}$ can be computed by a fast algorithm [8]. The result is denoted by

$$
\left(h_{j}\right)_{j=0}^{N}:=\frac{1}{N} C_{N+1}\left(d_{k}\right)_{k=0}^{N} .
$$

Note that $h_{N}=0$. Thus we get the following system of nonlinear equations

$$
\begin{aligned}
c_{0}^{4}+c_{1}^{4}+\ldots+c_{N-1}^{4} & =h_{0}, \\
c_{0}^{2} c_{1}^{2}+c_{1}^{2} c_{2}^{2}+\ldots+c_{N-2}^{2} c_{N-1}^{2} & =h_{1},
\end{aligned}
$$




$$
\begin{array}{ll}
c_{0}^{2} c_{2}^{2}+c_{1}^{2} c_{3}^{2}+\ldots+c_{N-3}^{2} c_{N-1}^{2} & =h_{2}, \\
& \vdots \\
c_{0}^{2} c_{N-1}^{2} & =h_{N-1}, \\
0 & =h_{N} .
\end{array}
$$

From (18) it follows that

$$
h_{0}=\frac{1}{N}\left(\frac{1}{2} d_{0}+\sum_{k=1}^{N-1} d_{k}+\frac{1}{2} d_{N}\right) .
$$

By (16) we have $c_{n}^{2}=f(n)(n=0, \ldots, N-1)$ such that the relation

$$
c_{0}^{4}+c_{1}^{4}+\ldots+c_{N-1}^{4}=h_{0}
$$

can be considered as a discrete Parseval equation for the vectors $(f(n))_{n=0}^{N-1}$ and $\left(\hat{f}\left(v_{k}\right)\right)_{k=0}^{N}$ :

$$
\sum_{n=0}^{N-1}(f(n))^{2}=\frac{1}{2 N}\left(|\hat{f}(0)|^{2}+2 \sum_{k=1}^{N-1} \frac{\left|\hat{f}\left(v_{k}\right)\right|^{2}}{\left(\operatorname{sinc} \frac{v_{k}}{2}\right)^{4}}+\frac{\pi^{4}}{16}|\hat{f}(\pi)|^{2}\right) .
$$

From (19), it follows immediately that all values $|f(n)|$ are bounded by the constant

$$
b:=\frac{1}{\sqrt{2 N}}\left(|\hat{f}(0)|^{2}+2 \sum_{k=1}^{N-1} \frac{\left|\hat{f}\left(v_{k}\right)\right|^{2}}{\left(\operatorname{sinc} \frac{v_{k}}{2}\right)^{4}}+\frac{\pi^{4}}{16}|\hat{f}(\pi)|^{2}\right)^{1 / 2} .
$$

In order to compute the real unknowns $c_{n}(n=0, \ldots, N-1)$, we have to solve the following nonlinear system

$$
\begin{aligned}
c_{0}^{4}+c_{1}^{4}+\ldots+c_{N-1}^{4} & =h_{0}, \\
c_{0}^{2} c_{1}^{2}+c_{1}^{2} c_{2}^{2}+\ldots+c_{N-2}^{2} c_{N-1}^{2} & =h_{1}, \\
c_{0}^{2} c_{2}^{2}+c_{1}^{2} c_{3}^{2}+\ldots+c_{N-3}^{2} c_{N-1}^{2} & =h_{2}, \\
& \vdots \\
c_{0}^{2} c_{N-1}^{2} & =h_{N-1} .
\end{aligned}
$$

Introducing the vector $\mathbf{h}:=\left(h_{n}\right)_{n=0}^{N-1}$, we obtain the nonlinear problem

$$
F_{\mathrm{c}}(\mathbf{z})=\mathbf{h}
$$

with $F_{\mathrm{c}}(\mathbf{z}):=T\left(\mathbf{z}^{2}\right) \mathbf{z}^{2}$, where $\mathbf{z}^{2}:=\left(c_{n}^{2}\right)_{n=0}^{N-1}$ is the pointwise square of $\mathbf{z}=\left(c_{n}\right)_{n=0}^{N-1}$ and $T\left(\mathbf{z}^{2}\right)$ is the Toeplitz matrix introduced in Section 2. Since the data vector $\mathbf{h}$ is based on measurements, we consider instead of the above nonlinear system the following least squares problem

$$
\left\|F_{\mathrm{c}}(\mathbf{z})-\mathbf{h}\right\|^{2}=\min .
$$


The inverse problem (21) is ill-posed. To cope with the ill-posedness, problem (21) has to be regularized. A known method is Tikhonov regularization [7]. Thus we determine an approximate minimizer of the Tikhonov functional

$$
\Phi_{\mathrm{c}}(\mathbf{z}):=\left\|F_{\mathrm{c}}(\mathbf{z})-\mathbf{h}\right\|^{2}+\alpha\|\mathbf{z}-\tilde{\mathbf{z}}\|^{2}
$$

as an approximate solution of the inverse problem (21), where $\alpha>0$ is a small regularization parameter and $\tilde{\mathbf{z}} \in \mathbb{R}^{N}$ is a coarse approximation of the solution. The choice of $\tilde{\mathbf{z}}$ is crucial. Available a priori information about the location of least squares solution of (22) has to enter into the selection of $\tilde{\mathbf{z}}$. Later we will construct $\tilde{\mathbf{z}}$ by multilevel strategy. By the choice of $\tilde{\mathbf{z}}$, we can influence which least squares solution we want to approximate. It can be shown that the Tikhonov functional (23) is locally a convex functional.

Now we can explicitly compute the Jacobian

$$
F_{\mathrm{c}}^{\prime}(\mathbf{z})=2\left(H\left(\mathbf{z}^{2}\right)+T\left(\mathbf{z}^{2}\right)\right) \operatorname{diag} \mathbf{z},
$$

where the Hankel matrix $H\left(\mathbf{z}^{2}\right)$ was explained in Section 2. Then there exists a vector $\mathbf{z}^{*} \in \mathbb{R}^{N}$ with

$$
\Phi_{\mathrm{c}}\left(\mathbf{z}^{*}\right)=\min \left\{\Phi_{\mathrm{c}}(\mathbf{z}) ; \mathbf{z} \in \mathbb{R}^{N}\right\} .
$$

Now we define a sequence $\left\{\mathbf{z}_{k}\right\}_{k=0}^{\infty}$ which converges to $\mathbf{z}^{*}$ for $k \rightarrow \infty$. Using the linear Taylor expansion

$$
F_{\mathrm{c}}(\mathbf{z}) \approx F_{\mathrm{c}}\left(\mathbf{z}_{k}\right)+F_{\mathrm{c}}^{\prime}\left(\mathbf{z}_{k}\right)\left(\mathbf{z}-\mathbf{z}_{k}\right)
$$

the simplified Tikhonov functional reads as follows

$$
\Phi_{\mathrm{c}}^{(k)}(\mathbf{z}):=\left\|F_{\mathrm{c}}\left(\mathbf{z}_{k}\right)+F_{\mathrm{c}}^{\prime}\left(\mathbf{z}_{k}\right)\left(\mathbf{z}-\mathbf{z}_{k}\right)-\mathbf{h}\right\|^{2}+\alpha\|\mathbf{z}-\tilde{\mathbf{z}}\|^{2} .
$$

This quadratic functional is minimized with the vanishing gradient condition

$$
\nabla \Phi_{\mathrm{c}}^{(k)}(\mathbf{z})=2 F_{\mathrm{c}}^{\prime}\left(\mathbf{z}_{k}\right)^{\mathrm{T}}\left[F_{\mathrm{c}}\left(\mathbf{z}_{k}\right)+F_{\mathrm{c}}^{\prime}\left(\mathbf{z}_{k}\right)\left(\mathbf{z}-\mathbf{z}_{k}\right)-\mathbf{h}\right]+2 \alpha(\mathbf{z}-\tilde{\mathbf{z}})=\mathbf{o} .
$$

Thus one ends up with the iterative Gauss-Newton method

$$
\mathbf{z}_{k+1}=\mathbf{z}_{k}-\Delta \mathbf{z}_{k} \quad(k=0,1, \ldots)
$$

with

$$
\left[F_{\mathrm{c}}^{\prime}\left(\mathbf{z}_{k}\right)^{\mathrm{T}} F_{\mathrm{c}}^{\prime}\left(\mathbf{z}_{k}\right)+\alpha I\right] \Delta \mathbf{z}_{k}=F_{\mathrm{c}}^{\prime}\left(\mathbf{z}_{k}\right)^{\mathrm{T}}\left(F_{\mathrm{c}}\left(\mathbf{z}_{k}\right)-\mathbf{h}\right)+\alpha\left(\mathbf{z}_{k}-\tilde{\mathbf{z}}\right) .
$$

This local Gauss-Newton method requires sufficiently good starting guesses $\mathbf{z}_{0}$, which we will construct by a multilevel strategy in Section 4. Analogously to (15), the linear system (26) has a unique solution for appropriate regularization parameter $\alpha>0$. 


\section{Multilevel strategy}

The multilevel strategy of the Gauss-Newton method is based on the following idea: In order to compute a solution of our least squares problem on a fine grid, first we compute the solution on a coarse grid. Then we interpolate the coarse grid solution to the fine grid and use it as initial guess of the local Gauss-Newton method on the fine grid.

The Gauss-Newton method is local quadratically convergent (see [9], p. 186) to an isolated minimizer of the Tikhonov functional $\Phi(\mathbf{y})$ and $\Phi_{\mathrm{c}}(\mathbf{z})$, respectively. The GaussNewton iteration requires a good initial guess. Therefore a multilevel strategy may be useful. We compute a first solution on a coarse grid. Further to the lower dimension of the nonlinear system for the coarse grid, an advantage of the multilevel strategy consists in a small number of critical points like extrema or saddle points in the minimization problem (8) and (22), respectively .

A well-known procedure for handling the phase retrieval problem is the GerchbergSaxton algorithm [10], which is a non-expansive fixed point algorithm. It is frequently convergent in practice, but it has not been proved to converge in all cases. Numerical experiments have shown that it requires up to $10^{4}$ or $10^{5}$ iterations in some cases [11]. These enormous numerical costs are closely related to the ill-posedness of the phase retrieval problem [12]. The multilevel strategy decreases the numerical effort by the computation of good approximations of the solution on coarse grids. Examples are given in Section 5.

Assume that the measurements $|f(n)|(n=0, \ldots, N-1)$ and $|\hat{f}(k \pi / N)|(k=$ $-N, \ldots, N-1)$ in Section 2 , and $|\hat{f}(k \pi / N)|(k=0, \ldots, N)$ in Section 3, respectively, are given. Additional values are not available. First, we explain the multilevel strategy for the constrained phase retrieval problem (21). The extensions needed to handle (6) subject to (7) analogously are given below.

\subsection{Multilevel strategy for the constrained phase retrieval problem}

Let $N=2^{J}$ with $J>3$ be given. By the multilevel strategy, we reduce the number of unknowns of (21). Here we use the special structure of the nonlinear system (21). By $\mathbf{z}_{j}=\left(c_{j, n}\right)_{n=0}^{2^{j}-1} \in \mathbb{R}^{2^{j}}\left(j=j_{0}, \ldots, J\right)$ we denote the solution of $F_{\mathrm{c}}\left(\mathbf{z}_{j}\right)=\mathbf{h}_{j}$ on the $j$-th grid $\left\{0,2^{J-j}, \ldots,\left(2^{j}-1\right) 2^{J-j}\right\}$. The right-hand side $\mathbf{h}$ is restricted to the $j$-th grid by

$$
\mathbf{h}_{j}=\left(h_{j, n}\right)_{n=0}^{2^{j}-1} \in \mathbb{R}^{2^{j}} \text { with } h_{j, n}:=2^{j-J} \cdot h_{2^{J-j} n}
$$

and $j=j_{0}, \ldots, J$. Note that $\mathbf{h}_{J}=\mathbf{h}$ and $\mathbf{z}_{J}=\mathbf{z}$. By (27), we select every $2^{J-j}$ th equation of (21). In addition to it, we group the $2^{J-j}$ unknowns $c_{2^{J-j} n}, \ldots, c_{2^{J-j}(n+1)-1}$ and replace them by $c_{j, n}$. So the factor $2^{j-J}$ comes into (27).

We introduce the linear spline function

$$
f_{j}(x):=\sum_{n=0}^{2^{j}-1} c_{j, n}^{2} \varphi\left(2^{j-J} x-n\right) \quad(x \in \mathbb{R}),
$$

which approximates $f$ from (16) on the $j$-th grid, see Figure 3 . The computation on the $j$-th grid is called the $j$-th level of the multilevel strategy. 
A solution $\mathbf{z}_{j}$ with $j<J$ of $F_{\mathrm{c}}\left(\mathbf{z}_{j}\right)=\mathbf{h}_{j}$ on the $j$-th grid of size $2^{j}$ approximates a solution $\mathbf{z}=\mathbf{z}_{J}$ of the system (21) on the fine $J$-th grid: The expression $\tilde{\mathbf{h}}_{j}:=$ $F_{\mathrm{c}}\left(\mathbf{z}_{j} \otimes \mathbf{1}_{J-j}\right) \in \mathbb{R}^{N}$ with $\mathbf{1}_{J-j}:=(1)_{k=0}^{2^{J-j}-1}$ and the Kronecker product $\otimes$ yields

$$
\tilde{h}_{j, 2^{J-j} n}=2^{J-j} h_{j, n}=h_{2^{J-j} n} \quad\left(n=0, \ldots, 2^{j}-1\right) .
$$

In general, the components $\tilde{h}_{j, m}$ and $h_{m}$ with $m \neq 2^{J-j} n$ do not coincide. Furthermore, the existence of a solution $\mathbf{z}^{*}$ of (21) does not assure the existence of coarse grid solutions $\mathbf{z}_{j}^{*}$ for $j<J$. In this case, the lower dimensional nonlinear systems are really nonlinear least squares problems.

From this approximation property we derive the following multilevel algorithm, starting on the $j_{0}-$ th grid.

\section{Algorithm 4.1 (Constrained phase retrieval problem)}

Input: $N=2^{J}, 3 \leq j_{0}<J,\left|\hat{f}\left(\frac{k \pi}{N}\right)\right|(k=0, \ldots, N), \alpha>0$.

0. Precompute $h_{n}(n=0, \ldots, N)$ by $(17)-(18)$.

1. For $j=j_{0}$ determine $\mathbf{z}_{j_{0}}^{*}$ as follows:

1.0. Choose a coarse approximation $\tilde{\mathbf{z}}_{j_{0}}$.

1.1. Determine $\mathbf{h}_{j_{0}}$ by (27).

1.2. Minimize the Tikhonov functional $\Phi_{\mathrm{c}}\left(\mathbf{z}_{j_{0}}\right)$ by the Gauss-Newton method (25) - (26) with the initial guess $\mathbf{z}_{j_{0}, 0}=\tilde{\mathbf{z}}_{j_{0}}$ and the coarse approximation $\tilde{\mathbf{z}}_{j_{0}}$. Use e.g. NLSQ-ERR from [9], pp. 219, 417. One gets the minimizer $\mathbf{z}_{j_{0}}^{*}$.

2. For $j=j_{0}+1: J$ compute $\mathbf{z}_{j}^{*}$ as follows:

2.0. Determine the coarse approximation $\tilde{\mathbf{z}}_{j}=\left(\tilde{z}_{j, k}\right)_{k=0}^{2^{j}-1}$ from $\mathbf{z}_{j-1}^{*}=\left(z_{j-1, k}^{*}\right)_{k=0}^{2^{j-1}-1}$ by $\tilde{z}_{j, 2 k}:=z_{j-1, k}^{*}\left(k=0, \ldots, 2^{j-1}-1\right)$ and by $\tilde{z}_{j, 2 k+1}:=\frac{1}{2}\left(z_{j-1, k}^{*}+z_{j-1, k+1}^{*}\right)$ $\left(k=0, \ldots, 2^{j-1}-1\right)$ with $z_{j-1,2^{j-1}}^{*}:=0$.

2.1. Compute $\mathbf{h}_{j}$ by $(27)$.

2.2. Minimize the Tikhonov functional $\Phi_{\mathrm{c}}\left(\mathbf{z}_{j}\right)$ by the Gauss-Newton method (25) - (26) with the initial guess $\mathbf{z}_{j, 0}=\tilde{\mathbf{z}}_{j}$ and the coarse approximation $\tilde{\mathbf{z}}_{j}$. Use e.g. NLSQ-ERR from [9], pp. 219, 417. One gets the minimizer $\mathbf{z}_{j}^{*}$.

Output: $\mathbf{z}^{*}=\mathbf{z}_{J}^{*}$.

Finally, a minimizer $\mathbf{z}^{*}$ of the Tikhonov functional (23) is found. Note that a coarse grid with 4 or even 8 nodes is often useless, since there are not enough points to construct a good approximative solution. Therefore we start with a coarse grid for $j_{0} \geq 3$. By (20), we know an upper bound $b$ of $|f(n)|(n=0, \ldots, N-1)$. Thus in the case $j_{0}=3$, we can choose $\tilde{\mathbf{z}}_{3}=\frac{\sqrt{b}}{4}(1,2,3,4,4,3,2,1)^{\mathrm{T}}$ as coarse approximation.

Damping strategies (see [9], p. 120) for the Gauss-Newton methods are recommendable in particular in the step 1.2, when the coarse approximation $\tilde{\mathbf{z}}_{j_{0}}$ is probably remote from the solution $\mathbf{z}_{j_{0}}^{*}$. 


\subsection{Multilevel strategy for the phase retrieval problem}

While solving the system (6) under the additional constraints (7) we are faced to a continuous manifold of solutions. These solutions are not isolated. With each solution $\mathbf{y}=\left(\mathbf{a}^{\mathrm{T}}, \mathbf{b}^{\mathrm{T}}\right)^{\mathrm{T}}$, the vector $\left(\mathbf{a}^{\mathrm{T}} \cos \alpha-\mathbf{b}^{\mathrm{T}} \sin \alpha, \mathbf{a}^{\mathrm{T}} \sin \alpha+\mathbf{b}^{\mathrm{T}} \cos \alpha\right)^{\mathrm{T}}$ for arbitrary $\alpha \in[0,2 \pi)$ is a solution, too. That corresponds to the replacement of $c_{n}$ by $c_{n} \mathrm{e}^{\mathrm{i} \alpha}$ in (1).

An opportunity to cope of this continuous manifold is scaling one of the components. Another method is Tikhonov regularization (12) which selects a particular solution out off the manifold.

The nonlinear system (6) subject to (7) has an approximation property which is analogous to that in Subsection 4.1. We solve $F(\mathbf{y})=\mathbf{g}$ on a stepwise refining grid. Again analogously to (27), we define $\mathbf{g}_{j}=\left(g_{j, n}\right)_{n=0}^{3 \cdot 2^{j}-1}$ by

$$
g_{j, n}:= \begin{cases}2^{j-J} \cdot g_{2^{J-j} n} & n=0, \ldots, 2^{j+1}-1, \\ g_{2^{J-j} n} & n=2^{j+1}, \ldots, 3 \cdot 2^{j}-1 .\end{cases}
$$

The latter restrictions without the factor $2^{j-J}$ concern the constraint conditions (7).

Now the solution $\mathbf{y}_{j}=\left(\mathbf{a}_{j}^{\mathrm{T}}, \mathbf{b}_{j}^{\mathrm{T}}\right)^{\mathrm{T}} \in \mathbb{R}^{2^{j+1}}$ of $F\left(\mathbf{y}_{j}\right)=\mathbf{g}_{j} \in \mathbb{R}^{3 \cdot 2^{j}}$ has the approximative property that $\tilde{\mathbf{g}}_{j}:=F\left(\mathbf{y}_{j} \otimes \mathbf{1}_{J-j}\right) \in \mathbb{R}^{3 N}$ fulfils

$$
\tilde{g}_{j, 2^{J-j} n}=g_{2^{J-j} n} \quad\left(n=0, \ldots, 3 \cdot 2^{j}-1\right) .
$$

Again, the linear spline functions

$$
f_{j}(x):=\sum_{n=0}^{2^{j}-1}\left(a_{j, n}+\mathrm{i} b_{j, n}\right) \varphi\left(2^{j-J} x-n\right) \quad(x \in \mathbb{R})
$$

approximate the function $f$ on the coarser grids. Hence, we get the following multilevel algorithm.

\section{Algorithm 4.2 (Phase retrieval problem)}

Input: $N=2^{J}, 3 \leq j_{0}<J, \mu \geq 1,|f(n)|(n=0, \ldots, N-1),\left|\hat{f}\left(\frac{k \pi}{N}\right)\right|(k=$ $-N, \ldots, N-1), \alpha>0$.

0 . Precompute $h_{n}(n=0, \ldots, 2 N-1)$ by $(2)-(5)$.

1. For $j=j_{0}$ determine $\mathbf{y}_{j_{0}}^{*}$ as follows:

1.0. Choose a coarse approximation $\tilde{\mathbf{y}}_{j_{0}}$.

1.1. Determine $\mathbf{g}_{j_{0}}$ by (29).

1.2. Minimize the Tikhonov functional $\Phi\left(\mathbf{y}_{j_{0}}\right)$ by the Gauss-Newton method (14) - (15) with the initial guess $\mathbf{y}_{j_{0}, 0}=\tilde{\mathbf{y}}_{j_{0}}$ and the coarse approximation $\tilde{\mathbf{y}}_{j_{0}}$. Use e.g. NLSQ-ERR from [9], pp. 219, 417. One gets the minimizer $\mathbf{y}_{j_{0}}^{*}$.

2. For $j=j_{0}+1: J$ compute $\mathbf{y}_{j}^{*}$ as follows:

2.0. Determine the coarse approximation $\tilde{\mathbf{y}}_{j}=\left(\tilde{y}_{j, k}\right)_{k=0}^{2^{j+1}-1}$ from $\mathbf{y}_{j-1}^{*}=\left(y_{j-1, k}^{*}\right)_{k=0}^{2^{j}-1}$ by $\tilde{y}_{j, 2 k}:=y_{j-1, k}^{*}\left(k=0, \ldots, 2^{j}-1\right)$ and by $\tilde{y}_{j, 2 k+1}:=\frac{1}{2}\left(y_{j-1, k}^{*}+y_{j-1, k+1}^{*}\right)$ $\left(k=0, \ldots, 2^{j}-1\right)$ with $y_{j-1,2^{j}}^{*}:=0$. 

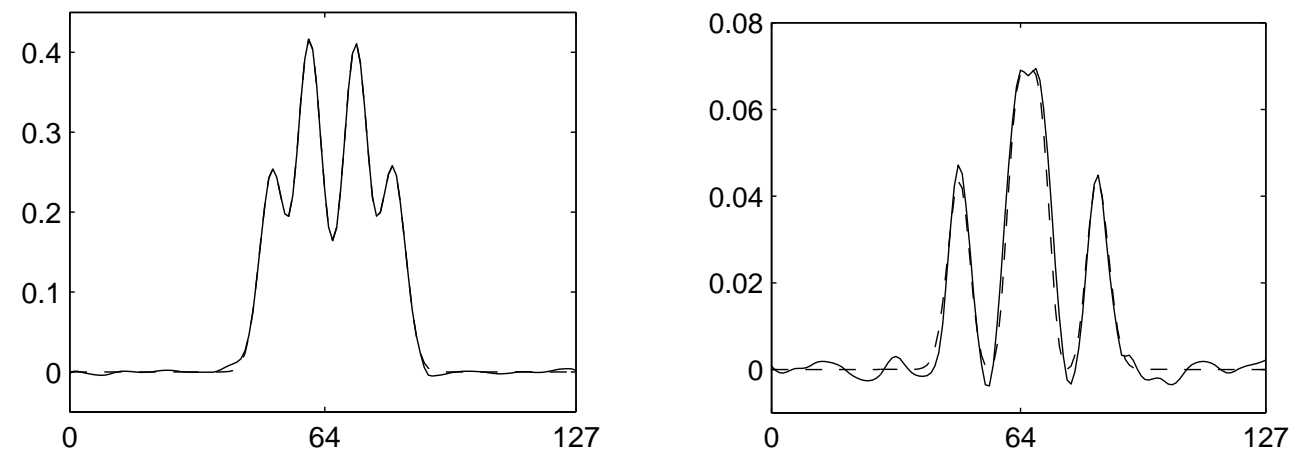

Figure 1. Real part $\mathbf{a}_{J}$ (left) and imaginary part $\mathbf{b}_{J}$ (right) of the computed solution (solid line) and the exact solution (dashed line) for the phase retrieval problem.

2.1. Compute $\mathbf{g}_{j}$ by (29).

2.2. Minimize the Tikhonov functional $\Phi\left(\mathbf{y}_{j}\right)$ by the Gauss-Newton method (14)(15) with the initial guess $\mathbf{y}_{j, 0}=\tilde{\mathbf{y}}_{j}$ and the coarse approximation $\tilde{\mathbf{y}}_{j}$. Use e.g. NLSQ-ERR from [9], pp. 219, 417. One gets the minimizer $\mathbf{y}_{j}^{*}$.

Output: $\mathbf{y}^{*}:=\mathbf{y}_{J}^{*}$.

Finally, a minimizer $\mathbf{y}^{*}$ of the Tikhonov functional (12) is found. The coarse approximation $\tilde{\mathbf{y}}_{j_{0}}$ can be chosen as follows $y_{j_{0}, k}=\left|f\left(2^{J-j_{0}} k\right)\right|$ for $k=0, \ldots 2^{j_{0}}-1$ and $y_{j_{0}, k}=0$ for $k=2^{j_{0}}, \ldots, 2^{j_{0}+1}-1$.

\section{Numerical tests}

\subsection{Tests for the phase retrieval problem}

We have tested the multilevel Algorithm 4.2 with numerous realistic examples. Therefore, we have chosen functions $f$ and hence $c_{n}=f(n) \in \mathbb{C}(n=0, \ldots, N-1)$. Using $a_{n}=\operatorname{Re} c_{n}$ and $b_{n}=\operatorname{Im} c_{n}$, we have generated the right-hand side $\mathbf{h}$ from (6) and $\mathbf{f}$ from (7), respectively. The true solutions $\mathbf{a}$ and $\mathbf{b}$ are plotted in Figure 1 as dashed linear splines over grid points $0, \ldots, N-1$ with $N=128$. In the present example, we use the function values

$$
f(n)=\left(\cos ^{2}\left(\frac{n}{7}\right)+0.2\right)\left(\mathrm{e}^{\kappa(n-56)^{2}}+\mathrm{e}^{\kappa(n-76)^{2}}\right)
$$

with $\kappa=-0.026+\mathrm{i} 0.004$ and $n=0, \ldots, 127$.

Then approximative solutions are computed using the multilevel Algorithm 4.2. The results are given as solid lines in Figure 1. Figure 2 shows the real and imaginary part of the differences between the exact and computed solution. Since the Gauss-Newton method is local quadratically convergent in this cases, an further improvement of the computed solution depends only on the tolerance used in the abortion criterium. Here, we get $\left\|F\left(\mathbf{y}_{J}^{*}\right)-\mathbf{g}\right\| \approx 4.6 \cdot 10^{-4}$.

With the penalty parameter $\mu=1$ and the regularization parameter $\alpha=0.01$, the 

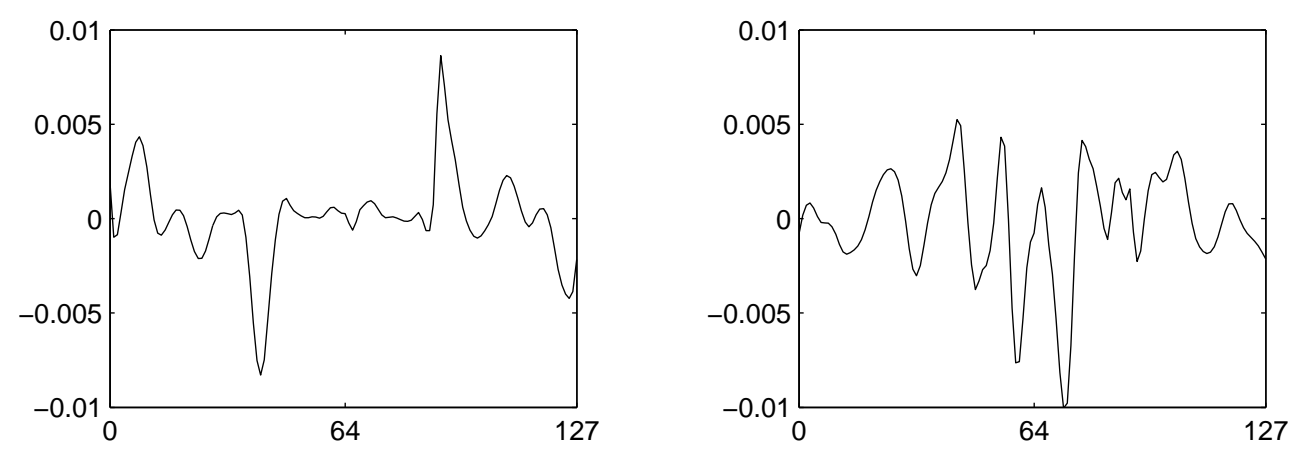

Figure 2. Difference between computed and exact solution, real part (left) and imaginary part (right).

Algorithm 4.2 requires 12 iteration steps for the computation of $\mathbf{y}_{3}^{*}$ on the initial level $j_{0}=3$, then 16 iterations for $\mathbf{y}_{4}^{*}$ on the fourth level, 19 steps for the fifth level, 12 steps for $j=6$ and 12 steps for the finest level $J=7$ with $N=128$. We see that good initial guesses for the Gauss-Newton method on fine grids yield to small numbers of iterations, in particular for the fine levels.

The right plot in Figure 1 shows additional small oscillations and a numerical artefact of the computed solution, where the true solution is already vanishing. It has been observed that the approximation of small components of the solution is worse than the one of large components. But, the artefacts in the region where $|f(n)|<\varepsilon$, i.e. outside of a relevant support, may be suppressed by just setting the components $a_{n}$ and $b_{n}$ to zero. The small oscillations can be faded out by smoothing terms or by a large number of damped Gauss-Newton steps.

Furthermore, the Algorithm 4.2 cannot work well for highly oscillating functions, because there are not enough sampling points to reconstruct highly oscillating functions on a coarser grid. Starting with $j_{0}=4$ or $j_{0}=5$ would provide useless approximative solutions on coarse grids, and one would need a lot of expensive Gauss-Newton steps in fine levels.

The Gauss-Newton algorithms NLSQ-RES and NLSQ-ERR from [9], pp. 416-417 has been tested with the above examples, too. Both algorithms failed to converge, when these algorithms were applied to the whole system (6) subject to (7) for $N=128$ for all tested nontrivial initial vectors and parameters. Otherwise, an application of these algorithms in each level was successful. This is a way to optimize the total numerical effort. This demonstrates the importance of the used multilevel strategy.

\subsection{Tests for the constrained phase retrieval problem}

The procedure to test the multilevel Algorithm 4.1 for the constrained phase retrieval problem is similar. Again realistic examples for $N=128$ has been tested, where the algorithms NLSQ-RES and NLSQ-ERR from [9], pp. 416-417 fail to converge. In the 

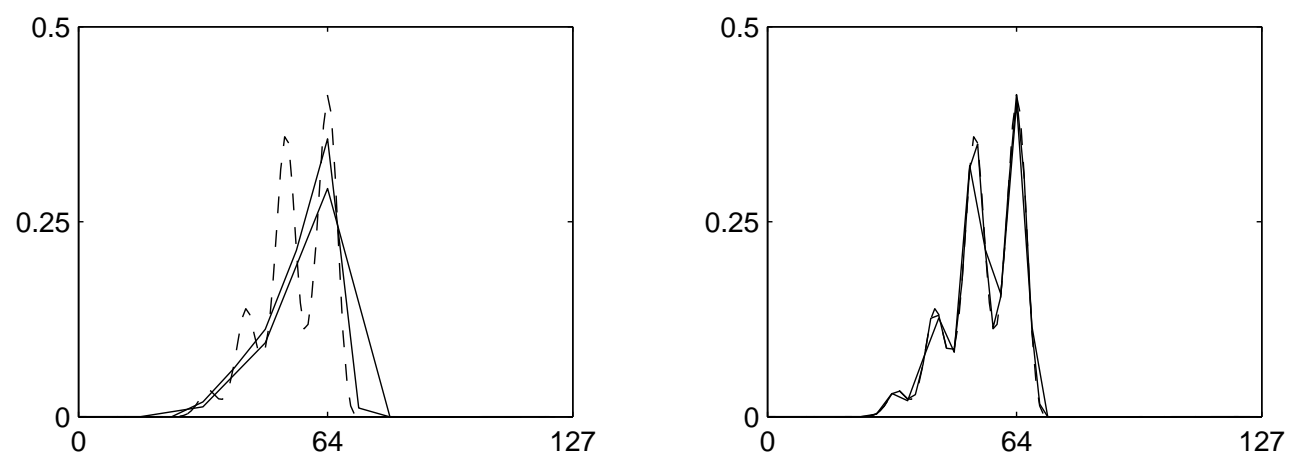

Figure 3. Intermediate solutions (solid lines) $f_{3}, f_{4}$ (left) and $f_{5}, f_{6}$ (right) while proceeding the multilevel-strategy, final computed solution $f$ (dashed line) for comparison.

following example, we use the function values

$$
f(n)=\left(\cos ^{2}\left(\frac{n}{7}\right)+0.2\right)\left(\mathrm{e}^{-0.026(n-56)^{2}}+1.5 \mathrm{e}^{-0.02(n-76)^{2}}\right)
$$

with $n=0, \ldots, 127$. Figure 3 gives the intermediate solutions $f_{3}, \ldots, f_{6}$ and the computed solution $f$ for comparison. In the left plot the coarse grid solutions are clearly visible, they are linear splines of larger step sizes. The intermediate solutions $f_{3}$ and $f_{4}$ are shown for demonstration, they are not necessary in practical applications of the Algorithm 4.1.

In general, the nonlinear problem (21) has various feasible solutions. First, if $\mathbf{z}=$ $\left(c_{n}\right)_{n=0}^{N-1}$ is a solution of $(21)$, then the reflected vector $\left(c_{N-1-n}\right)_{n=0}^{N-1}$ is a solution, too. Furthermore, there are nontrivial distinct solutions. For instance, if $\mathbf{h}=(22,14,6,1)^{\mathrm{T}}$, then feasible solutions of $(21)$ are $\mathbf{z}=(1,2,4,1)^{\mathrm{T}},(1.874,1.648,1.229,0.534)^{\mathrm{T}}$ and their reflections.

Figure 4 shows two different solutions $f$ and $g$ of (21), which are not reflections of each other. The difference

$$
\delta(k):=\left|\hat{f}\left(\frac{k \pi}{128}\right)\right|-\left|\hat{g}\left(\frac{k \pi}{128}\right)\right| \quad(k=0, \ldots, 127)
$$

is shown in the right plot. The difference is relatively small for $k \in[0,63]$, where the Fourier transform itself has a non-negligible modulus. For larger $k$, the Fourier transform is nearly vanishing and the computed difference is of the magnitude $10^{-4}$.

The observations about the numerical behavior are similar to the ones of the unconstrained phase retrieval problem. In particular, highly oscillating functions complicate the numerical solution. But already due to the lower dimension and due the fact that in most observed examples the nonlinear system (21) has actual solutions even in the coarser levels, the constrained phase retrieval problem has a better numerical nature than the unconstrained one.

Problems due to active unilateral constraints $c_{j, n}=0$ have not been observed, cf. [9], p. 188. A loss of convergence rate occurs for very small $c_{j, n}$ and does not influence the approximation of the searched function $f$. Alternatively, constrained optimization 

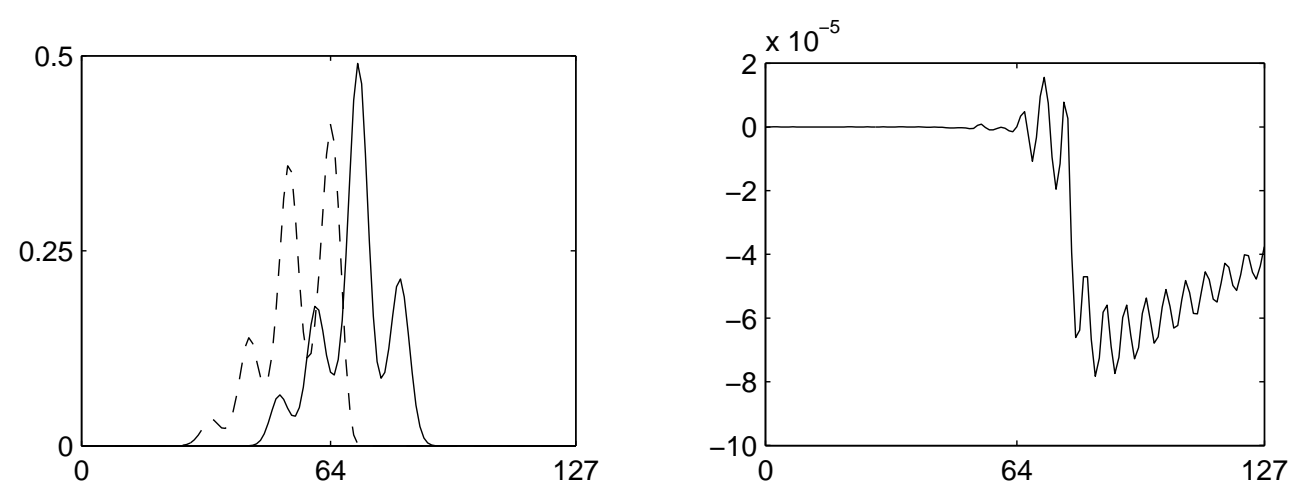

Figure 4. Two distinct computed solutions $f$ and $g$ for one right-hand side $\mathbf{h}$ (left) and the difference $\delta$ defined by (30) (right).

software may be used.

Nevertheless, both phase retrieval problems are still ill-posed [12], and generally speaking, the ill-posedness is weakened by the multilevel strategy.

\section{Conclusions}

Two new phase retrieval algorithms for different one-dimensional phase problems have been developed and tested with numerous realistic examples. These new algorithms are robust, converge quickly and should be useful in a variety of physically important problems. The obtained significant increase in speed could be used for real time phase retrieval in laser optics (e.g. FROG and TASC [3]).

\section{Acknowledgment}

We are grateful for the financial support we received from the Deutsche Forschungsgemeinschaft (DFG) through the DFG researcher group "Quantum Optics in Semiconductor Nanostructures" (FOR 485). Further we thank the referees for their helpful comments.

\section{References}

[1] Trebino R 2002, Frequency-Resolved Optical Gating: The Measurement of Ultrashort Laser Pulses (Dordrecht: Kluwer Acad. Publ. Group)

[2] Wong V and Walmsley I A 1997 Ultrashort-pulse characterization from dynamic spectrograms by iterative phase retrieval J. Opt. Soc. Am. B 14 944-949

[3] Seifert B, Stolz H and Tasche M 2004 Nontrivial ambiguities for blind frequency-resolved optical gating and the problem of uniqueness J. Opt. Soc. Am. B 21 1089-1097

[4] Webster G and Hilgenfeld R 2002 Perspectives on single molecule diffraction using the X-ray free electron laser Single Molecules 3 61-66

[5] Klibanov M V, Sacks P E and Tikhonravov A V 1995 The phase retrieval problem Inverse Problems 11 1-28 
[6] Van Loan C F 1992 Computational Framework for the Fast Fourier Transform (Philadelphia: SIAM)

[7] Engl H W, Kunisch K and Neubauer A 1989 Convergence rates for Tikhonov regularisation of non-linear ill-posed problems Inverse Problems 5 523-540

[8] Plonka G and Tasche M 2005 Fast and numerically stable algorithms for discrete cosine transforms Linear Algebra Appl. 394 309-345

[9] Deuflhard P 2004 Newton Methods for Nonlinear Problems (Berlin: Springer)

[10] Gerchberg R W and Saxton W O 1972 A practical algorithm for the determination of phase from image and diffraction plane pictures Optik 35 237-246

[11] Fienup J R 1982 Phase retrieval algorithms: a comparison Appl. Optics 21 2758-2769

[12] Blaschke-Kaltenbacher B and Engl H W 1997 Regularization methods for nonlinear ill-posed problems with applications to phase reconstruction, In: Inverse Problems in Medical Imaging and Nondestructive Testing, Engl H W, Louis A K and Rundell W (Wien: Springer) 17-35 